\title{
The E3 Ligase RNF34 is a Novel Negative Regulator of the NOD1 Pathway
}

\author{
Rui Zhang $^{\text {a }}$ Jian Zhao ${ }^{\text {Yuhua Song }}{ }^{\mathrm{b}}$ Xu Wang ${ }^{\mathrm{a}}$ Lili Wang $^{\mathrm{a}}$ Jian Xu Chun Song $^{\mathrm{a}}$ \\ Fang Liua
}

aDepartment of Colorectal Surgery, Liaoning Cancer Hospital \& Institute, Shenyang, ${ }^{\text {'The Breast Center }}$ of the Affiliated Hospital of Qingdao University, Qingdao, P.R. China

\author{
Key Words \\ $\mathrm{NOD} 1 \cdot \mathrm{RNF34} \cdot \mathrm{NF}-\mathrm{\kappa B}$
}

\begin{abstract}
Background/Aims: To identify the regulator of nucleotide-binding oligomerization domaincontaining protein 1 (NOD1) and its regulatory function. Methods and Results: We performed a yeast two-hybrid screening assay and identified the E3 ligase RNF34 as a candidate partner of NOD1. Using co-immunoprecipitation (Co-IP) and glutathione $S$ transferase (GST)-pull down assays, we further confirmed that RNF34 is associated with NOD1. Western blotting showed that RNF34 downregulated the stability of NOD1 and promoted its ubiquitination. Functional analysis demonstrated that RNF34 overexpression inhibited NOD1-dependent activation of nuclear factor-kappa B (NF-kB), whereas knockdown of RNF34 using small interfering RNA increased NF-KB activation following stimulation from NOD1 overexpression or transfection of $\gamma$-D-glutamyl-meso-diaminopimelic acid. Conclusion: These findings confirm that RNF34 is a negative regulator of the NOD1 pathway through direct interaction and ubiquitination of NOD1, and suggest a novel regulatory mechanism of NOD1.
\end{abstract}

Copyright $(2014$ S. Karger AG, Basel

\section{Introduction}

The innate immune system is the first line of defense against invading organisms, in which recognition of a large variety of microorganisms and their molecules occurs via specific pattern recognition receptors (PRRs) present on the cell surface or in the cytosolic compartment of both innate immune and non-immune cells [1]. There are several families of PRRs including membrane-associated Toll-like receptors, cytosolic nucleotide-binding

Chun Song,

and Dr. Fang Liu
Department of Colorectal Surgery, Liaoning Cancer Hospital \& Institute, Shenyang, Liaoning Province 110042 (P.R. China)

E-Mail chunsong163@163.com and E-Mail liufang_work@sina.com 
oligomerization domain-containing protein (NOD)-like receptors (NLRs), RIG-I-like receptors, and AIM2-like receptors [2]. NOD1 is a member of the NLR family and recognizes specific bacterial molecules, $\Upsilon$-D-glutamyl-meso-diaminopilemic acids, derived from peptidoglycans found primarily in Gram-negative bacteria [3]. Upon ligand binding, the NOD domain undergoes homo-oligomerization, followed by activation of downstream cascade signaling molecules through protein-protein interactions [4].

The activation of the mitogen-activated protein kinase and nuclear factor kappa B (NF$\mathrm{kB}$ ) pathways by NOD1 leads to cytokine, chemokine, antimicrobial peptide production, and, in some cells, apoptosis [5-7]. There is accumulating evidence that NOD1 mediates host defense responses to intracellular pathogens such as Shigella flexneri [8], enteroinvasive Escherichia coli [9], Campylobacter jejuni [10], Helicobacter pylori [11], and Salmonella enterica serotype typhimurium [12], suggesting the important role of NOD1 in host innate immunity. NOD1 is also involved in monitoring the integrity of the epithelial cytosol by promoting autophagy of invading pathogens [13]. Recent reports have expanded the role of NOD1 in also inducing apoptosis and controlling tumor cell growth; the activation of NOD1 resulted in caspase-8- and caspase-9-mediated cell death in different cell lines [14]. The absence of NOD1 was recently reported to be associated with breast tumor growth and increasing sensitivity to estrogen stimulation [15].

Insufficient activation of PRRs cannot protect cells from invading pathogens; however, enhanced levels of inflammation elicits cell and tissue damage [16]. Therefore, the activation of these PRRs must be tightly controlled. Much progress has been made recently with regards to understanding the regulation of several PRR pathways, but little is known about NOD1. To identify new regulators that might interact with NOD1, a yeast two-hybrid library was screened using NOD1 protein as bait. An E3 ligase, RNF34, was selected to interact with NOD1, and this association was confirmed by in vivo and in vitro interaction assays. Furthermore, we found that RNF34 increased the ubiquitination of NOD1 and decreased the expression of NOD1 in a proteasome-dependent manner. These data suggest that RNF34 is a novel E3 ligase of NOD1 that may play an important role in negative regulation of the NOD1 pathway.

\section{Materials and Methods}

\section{Yeast Two-Hybrid Screening}

The pGBKT7 (Clontech System 3) DNA-binding domain vector was used to express the NOD1 fragment (amino acids 1-465) containing the CARD and NBD domains. The human spleen cDNA library from Clontech was fused to the GAL4 activation domain in pACT2. Screens were conducted in the reporter yeast strain AH109 according to the manufacturer's instructions. Transformants were plated on synthetic medium lacking tryptophan, leucine, adenine, and histidine. Approximately three million transformants were screened. Then, pGBKT7-NOD1 and candidate clones were co-transfected to the yeast cells to verify the interaction between the candidates and the NOD1 bait. Yeast $\beta$-galactosidase activity, determined as the MEL1 gene (Alpha-galactosidase) response to GAL4 activation, was evaluated in plates containing X-gal (BD Biosciences; Clontech).

\section{Plasmids and Antibodies}

The RNF34 gene was amplified from RNF34-pACT2 that was screened from the yeast two-hybrid screening assay. The Flag-tagged and RNF34 expression plasmids were ligated into a pcDNA3 vector and pCMV-Myc plasmids (Clontech). Plasmids encoding glutathione S transferase (GST) fusion proteins were sub-cloned into the in-frame of pGEX-4T-1 (Amersham Pharmacia Biotech). The RNF34 (H342A) mutant was constructed by using a quick-change mutagenesis kit (Strategene Corp.). The reporter constructs NF$\kappa B-L u c$ and IL-8-Luc and the expression vectors for NOD1 and ubiquitin have been described previously [17]. All of the constructs were confirmed by DNA sequencing. 
Cell culture, Transfection, and RNA Interference (RNAi)

The human embryonic kidney cell line 293T and colon carcinoma cell line HT-29 were grown in Dulbecco's modified Eagle medium (supplemented with fetal bovine serum (Hyclone) and maintained at 37 ${ }^{\circ} \mathrm{C}$ under $5 \% \mathrm{CO}_{2}$ in a humidified incubator. For transfections, cells were plated to $85-90 \%$ confluency and transfected with Lipofectamine 2000 (Invitrogen).

Luciferase Reporter Assays

Cells were seeded into 24-well plates and transfected the following day using Lipofectamine 2000 with the NF- $\kappa B$ luciferase reporter (NF- $\kappa B-L u c, 100 \mathrm{ng}$ ) and pRL-TK (2 ng) together with plasmids encoding NOD1 and the Myc-vector, Myc-RNF34. After $18 \mathrm{~h}$, samples were lysed in passive lysis buffer and activity was measured using a dual-luciferase reporter assay system (Promega Corp.) as described previously[17]. RNA oligos targeting RNF34 or controls for RNAi were synthesized by Genepharm Corp. The RNAi sequence that targets RNF34 was CTCCGTTTGTTCAGTCTTA.

\section{Immunoprecipitation (IP), GST-pull Down, and Immunoblot Analysis}

For IP, extracts were lysed in lysis buffer (50 mM Tris- $\mathrm{HCl}$ (pH 8.0), $150 \mathrm{mM} \mathrm{NaCl}$, and 1\% NP-40 in the presence of Complete $\AA$ protease inhibitor cocktail; Roche Applied Science). For IPs involving over-expressed proteins in 293T cells, $0.5 \mathrm{mg}$ of total cell lysate was incubated with Flag beads for $2 \mathrm{~h}$ at $4{ }^{\circ} \mathrm{C}$ with constant shaking. For precipitations of endogenous proteins, $1 \mathrm{mg}$ of total cell lysates was immunoprecipitated by anti-NOD1 antibody and protein A/G Sepharose beads. For the GST-pull down assay, cell lysates containing Flag-NOD1 were incubated for $2 \mathrm{~h}$ at $4{ }^{\circ} \mathrm{C}$ with $2 \mu \mathrm{g}$ purified GST or GST-RNF34 fusion proteins bound to glutathione beads. The immunoprecipitates were washed with NP-40 lysis buffer and then subjected to immunoblotting analysis. Briefly, the immunoprecipitated proteins were separated by $8-12 \%$ sodium dodecyl sulfate-polyacrylamide gel electrophoresis (SDS-PAGE) and blotted onto polyvinyldifluoride membranes. The specific protein was detected with anti-Flag, anti-Myc (Protech), anti-RNF34 (Bethyl Laboratories, Inc.), anti-NOD1 (Santa Cruz Biotechnology), or anti-GAPDH (Peprotech Corp.) antibodies.

\section{Ubiquitination Assays}

293T cells were co-transfected with equal amounts of Flag-NOD1 and HA-ubiquitin, together with Myc-vector, Myc-RNF34, or Myc-RNF34 (H342A). Twenty-four hours later, the proteasome inhibitor MG132 $(10 \mu \mathrm{M})$ was added and incubated for $6 \mathrm{~h}$. Cell lysates were mixed with anti-Flag agarose and the IP was analyzed by immunoblotting with anti-HA antibody and Flag agarose.

\section{Statistics}

All experiments in this study were performed independently at least four times. Student's t-test was used for the comparison of four independent treatments. For all tests, a p value $<0.05$ was considered statistically significant.

\section{Results}

\section{RNF34 is a Novel Partner of NOD1}

To identify the proteins involved in NOD1 regulation, we screened a human mammary cDNA library using amino acids 1-465 of NOD1 as bait in the yeast two-hybrid system. From the $5 \times 10^{6}$ transfectants, three interacting clones were identified. By aligning sequences using National Center for Biotechnology Information (NCBI) blasting (http://blast.ncbi.nlm.nih. gov), the sequence of the clones was shown to be identical with that of RNF34. The specificity of this interaction was confirmed in a direct two-hybrid binding assay. Transformation of yeast cells with pACT2-RNF34 together with the pGBKT7 vector did not activate the reporter genes, suggesting specific interaction of RNF34 with NOD1 (Fig. 1A). To further confirm the interaction, co-IP was performed in 293T cells that had been transfected with Myc-RNF34 and Flag-NOD1 or Flag-vector. Myc-RNF34 was co-precipitated by anti-Flag agarose only in the presence of Flag-NOD1 (Fig. 1B). To demonstrate the interaction of NOD1 and RNF34 in vitro, GST and GST-tagged RNF34 (GST-RNF34) were purified and added to lysates from 293T cells expressing Flag-NOD1. Analysis of the absorbates by immunoblotting with anti- 


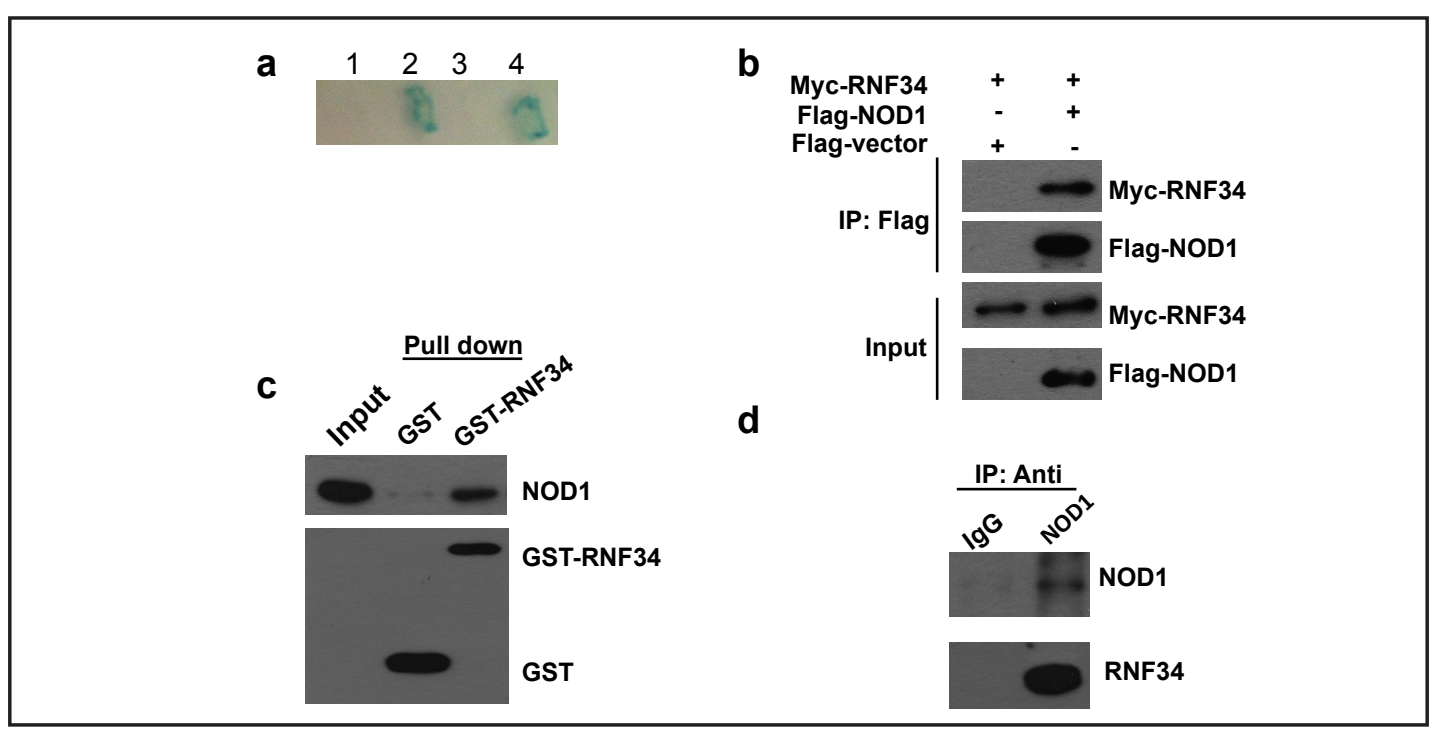

Fig. 1. NOD1 interacts with RNF34 in vivo and in vitro. a: Indicated plasmids were co-transected to yeast AH109 competent cells, and transformants were inoculated on synthetic medium (Try-, Leu-, Ade-, and His-) containing X-gal. 1, pGBKT7-NOD1+pGADT7; 2, pGBKT7-p53+ pGADT7-T; 3, pGBKT7+pGAD-RNF34; 4, pGBKT7-NOD1+pGAD-RNF34. b: The 293T cells were transfected with Flag-NOD1 and Myc-RNF34 expression plasmids. Twenty-four hours later, the cells were harvested and then lysed by NP-40 lysis buffer. Lysates were immunoprecipitated using anti-Flag beads. The absorbates and total lysates were separated in SDS-PAGE and analyzed by immunoblotting with anti-Flag and Myc antibody. c: The GST or GST-RNF34 fusion protein absorbates from cell lysates containing Flag-NOD1 were analyzed by immunoblotting with anti-Flag and anti-GST antibodies. d: HT-29 cells were lysed in RIPA buffer, and the lysates were immunoprecipitated using RNF34-specific antibody and normal IgG as a control. The immuno-complex was separated in SDSPAGE and analyzed by immunoblotting with anti-RNF34 and NOD1 antibody.

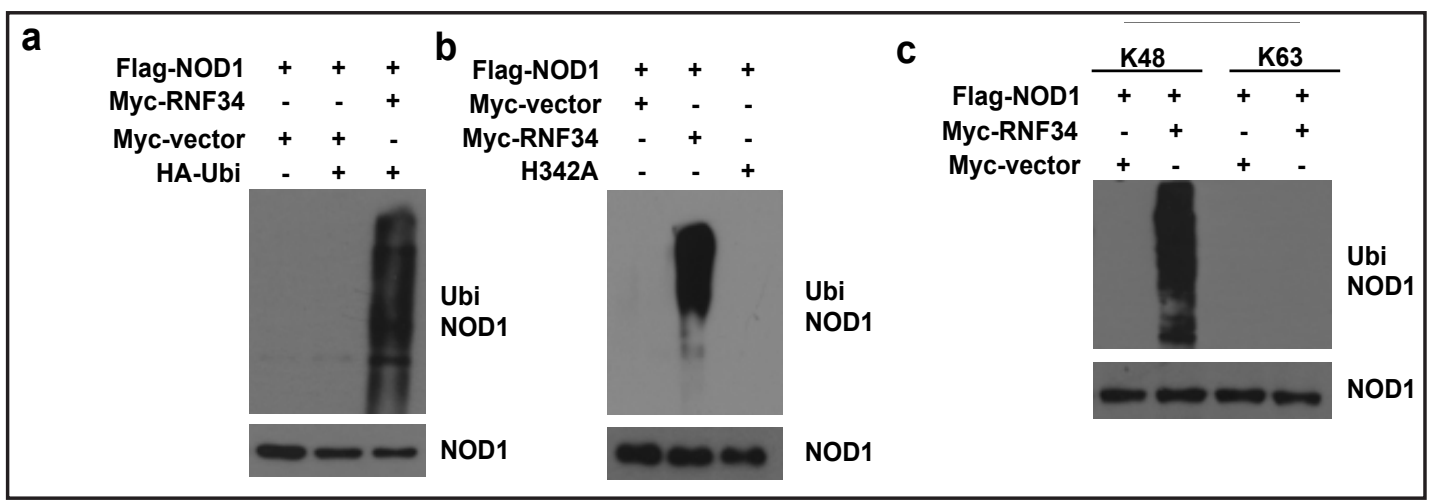

Fig. 2. RNF34 promotes the ubiquitination of NOD1. a: The 293T cells were transfected with Flag-NOD1 and HA-ubiquitin together with Myc-vector or Myc-RNF34 plasmids. Twenty-four hours later, MG132 was added to the supernatant for $6 \mathrm{~h}$. Cell lysates were mixed with anti-Flag agarose and the immunoprecipitation was analyzed by immunoblotting with anti-HA and Flag antibody. b: The 293T cells were transfected with Flag-NOD1 and HA-ubiquitin together with Myc-vector, Myc-RNF34, or Myc-RNF34 E3-dead mutant (H342A) expression plasmids. The absorbates enriched by Flag agarose were blotting using anti-HA and Flag antibody. c: The 293T cells were transfected with Flag-NOD1 and HA-ubiquitin (K48 or K63) together with Myc-vector or Myc-RNF34 plasmids. The absorbates were blotted using anti-HA and Flag antibody.

HA showed binding of NOD1 to GST-RNF34 (Fig. 1C). Furthermore, endogenous NOD1 and RNF34 were able to form a complex in HT-29 cells that expressed both NOD1 and RNF34 (Fig. 1D). These findings indicate that RNF34 is a novel binding protein of NOD1. 


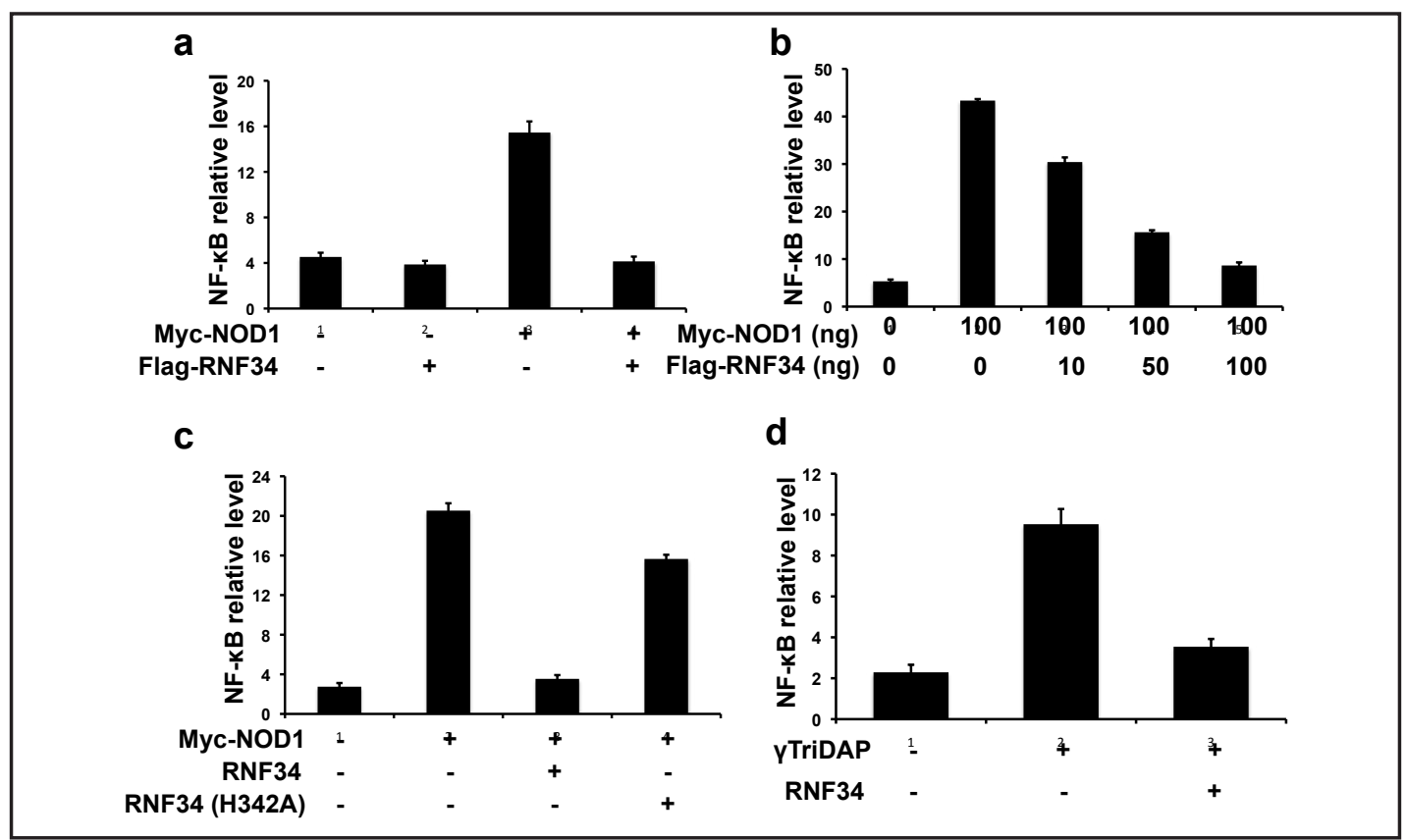

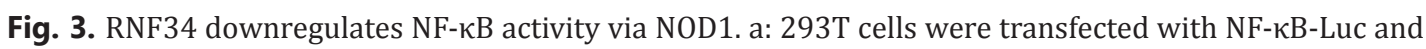
pRL together with Flag-NOD1 and Myc-vector or Myc-RNF34 plasmids. Twenty-four hours later, the cells were lysed in passive lysis buffer and activity of luciferase and Renilla were measured using a dual-luciferase reporter assay system. The error bars represent SDs from the mean values of triplicate experiments. b: The 293T cells were transfected with Flag-NOD1, increasing amounts of Myc-RNF34 plasmid as indicated, together with NF- $\kappa B$ luciferase and pRL plasmid. The luciferase activity was measured $24 \mathrm{~h}$ later and

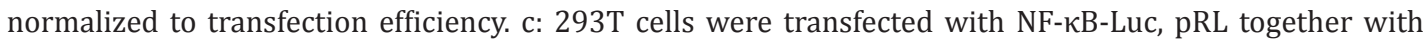
Flag-NOD1 and Myc-vector, Myc-RNF34, or Myc-RNF34 (H342A) plasmids. The relative activity of NF- $\mathrm{B}$ was assayed. d: HT-29 cells were transfected with Myc-RNF34, NF- $\kappa B$ luciferase, and pRL plasmids, and after18 $\mathrm{h}$, were stimulated with $\gamma$ TriDAP for an additional $24 \mathrm{~h}$. NF- $\kappa \mathrm{B}$ activation in response to NOD1 ligands was assayed in the absence or presence of RNF34.

\section{RNF34 Induces Increased Ubiquitination of NOD1}

Since RNF34 has been reported as an E3 ligase, we evaluated whether NOD1 is a substrate of RNF34. As shown in Fig. 2A, after IP of NOD1 with anti-Flag agarose, slowly migrating smears of the receptor were detected by using anti-HA antibodies, thus confirming that the level of NOD1 ubiquitination increased upon RNF34 overexpression. Mutation of a conserved zinc-binding histidine residue within the RING domain (H342A) abolished the E3 activity of RNF34 [18], indicating the necessity of an intact RING domain to mediate ubiquitination of NOD1 (Fig. 2B). We further analyzed the ligase classes of polyubiquitin chains supported by the E3 ligase activity of RNF34. Two mutant ubiquitins, in which lysine was only at position 48 or 63 and all the other lysines were substituted by arginine, together with NOD1 and RNF34 were transfected in 293T cells. As shown in Fig. 2C, RNF34 predominantly uses the lysine at position 48 on ubiquitin for the formation of a polyubiquitylation chain.

\section{RNF34 Decreases the Transactivational Activity of NOD1}

Next, we assessed whether RNF34 promotes NOD1 transactivation activity. First, we detected NF- $\mathrm{BB}$ by conducting a luciferase reporter assay in 293T cells. As shown in Fig. 3A, RNF34 decreased NF- $\kappa$ B-Luc activity in the presence of NOD1. With increasing RNF34, the transactivity of NOD1 was downregulated in a dose-dependent manner (Fig. 3B). When RNF34 mutants with defective E3 activity (H342A) were co-transfected with NOD1, the $\mathrm{NF}-\kappa \mathrm{B}$ failed to decrease in contrast to wild type RNF34, indicating that the E3 activity of RNF34 is necessary for regulating NOD1 (Fig. 3C). To examine the effects of ligands on NOD1 


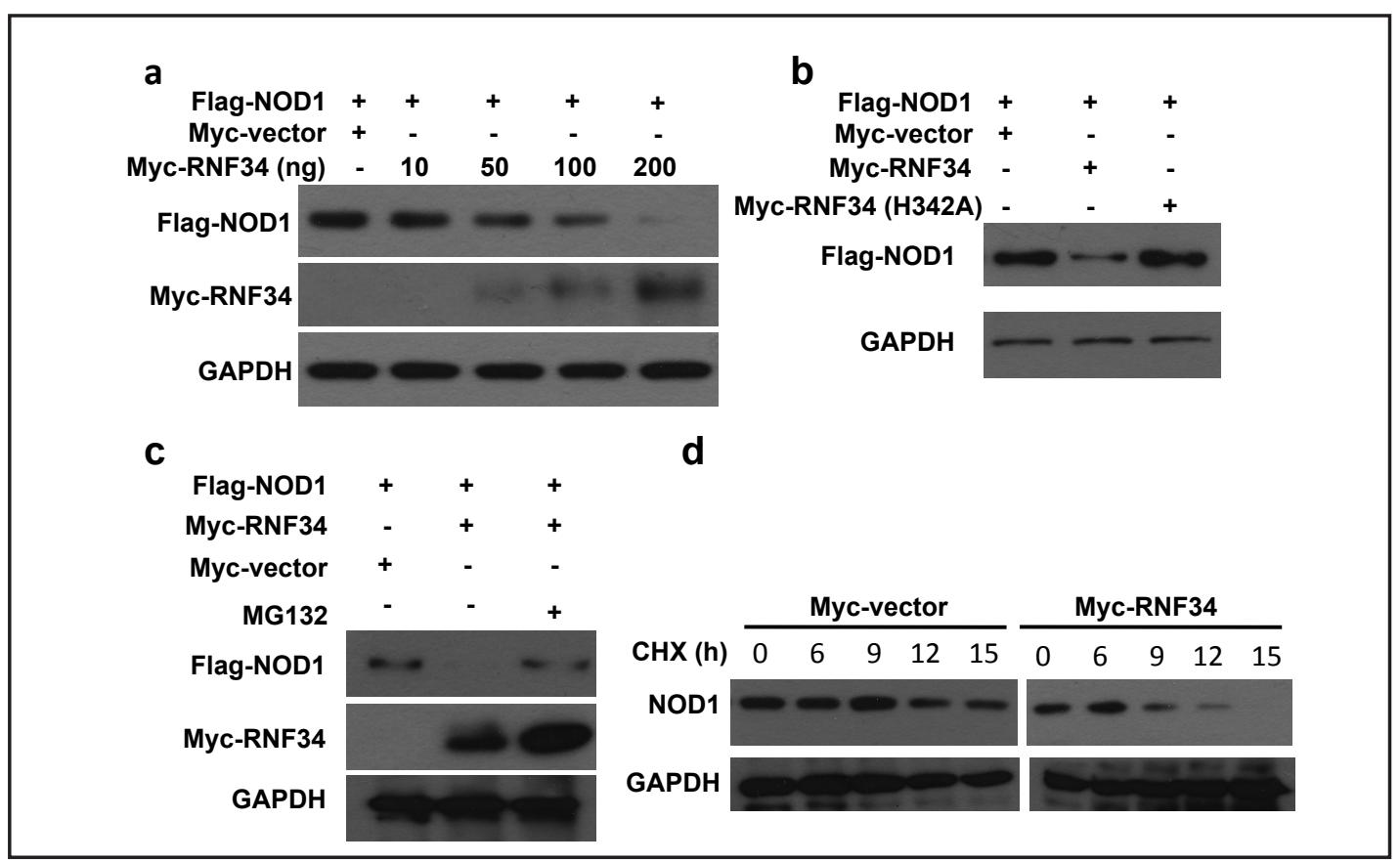

Fig. 4. RNF34 destabilizes NOD1. a: 293T cells were transfected with equal amounts of Flag-NOD1 and increasing amounts of Myc-RNF34 plasmids as indicated. Twenty-four hours later, the cell lysates were blotted using Flag and Myc antibodies. GAPDH was used as the equal loading control. b: 293T cells were transfected with NOD1 and RNF34 or H342A mutants. The expression of NOD1 was detected using Flag and Myc antibodies. c: 293T cells were transfected with equal amounts of Flag-NOD1 and Myc-RNF34 plasmids. After 24 h, MG132 or dimethyl sulfoxide was added to the culture medium. The expression of NOD1 and RNF34 was detected using Flag and Myc antibodies. d: 293T cells were transfected with the expression vector encoding Flag-NOD1 with or without RNF34. After $24 \mathrm{~h}$, cells were treated with cycloheximide (50 $\mathrm{mM}$ ). The expression level of NOD1 was monitored by immunoblotting using a Flag antibody.

transactivation by RNF34, HT-29 cells were treated with $\gamma$-D-glutamyl-meso-diaminopimelic acid ( $\gamma$ TriDAP). Fig. 3D shows that RNF34 decreased the transcriptional activity of NOD1 when the ligands were bound.

\section{RNF34 Leads to Proteasomal Degradation of NOD1}

The discovery that RNF34 contributes to ubiquitination of NOD1 suggested its involvement in the regulation of NOD1 stability through ubiquitin-mediated proteolysis. As shown in Fig. 4A, when increasing amounts of RNF34 expression vectors were transiently co-transfected with equal amounts of NOD1, the expression of NOD1 was significantly decreased. This effect of RNF34 on NOD1 stability was dependent on its E3 ligase activity (Fig. 4B). The RNF34-dependent downregulation of NOD1 was completely blocked by the proteasome inhibitor MG132, indicating that this effect is mediated by the ubiquitin/ proteasome pathway (Fig. 4C). We next measured the effect of RNF34 overexpression on the stability of NOD1 by conducting a pulse-chase experiment in HT-29 cells using cycloheximide as an inhibitor for protein synthesis. We also observed a significant decrease in the stability of NOD1 in the presence of RNF34 (Fig. 4D).

RNF34 Knockdown Promotes Activity and Stability of NOD1

To further confirm the effects of RNF34 on NOD1, we knocked down the expression of RNF34 using RNAi. RNF34-targeted small interfering RNA (siRNA) was transfected to HT29 cells, and as shown in Fig. 5A, RNF34 expression was knocked down significantly. When 


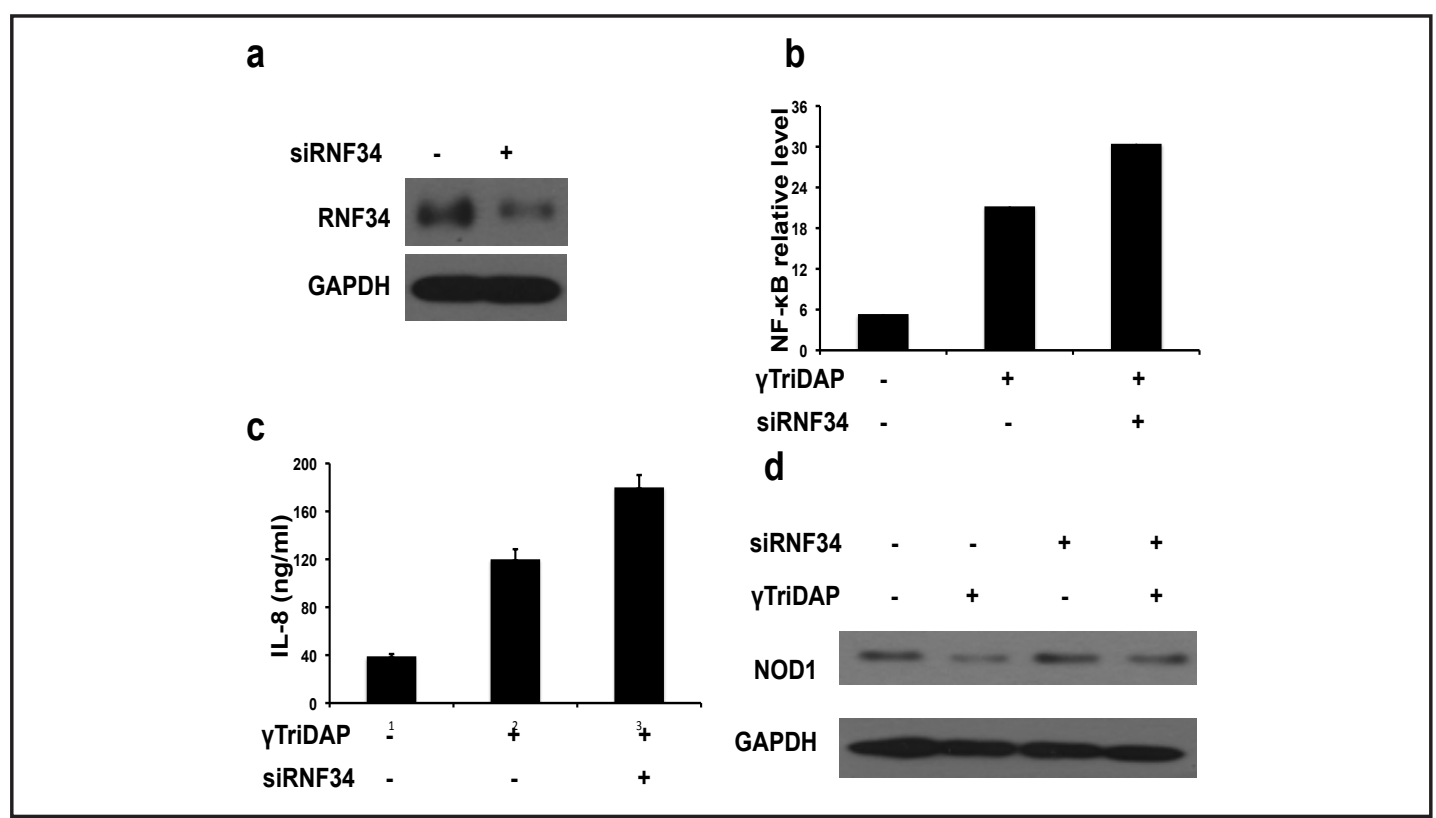

Fig. 5. Knockdown of RNF34 promotes stability and NF- $\kappa$ B activation via NOD1. a: HT29 cells were transfected with oligo RNAs targeting RNF34 or control RNA. To detect the efficiency of the knockdown, cell lysates were blotted with RNF34 and GAPDH antibodies. The cells were repeatedly transfected with oligo RNA targeting RNF34 or control RNA. Whole cell lysates were analyzed by immunoblotting with NOD1, RNF34, and GAPDH antibodies. b: HT29 cells were transfected with oligo RNA targeting RNF34 or control RNA. After 24 h, HT29 cells were transfected with siRNF34 or control siRNA together with NF- $\mathrm{BB}$ luciferase and pRL plasmid. After treatment with $\gamma$ TriDAP, the luciferase activity was measured and normalized to transfection efficiency. c: IL-8 levels in the supernatant obtained from (b) were measured by an enzyme-linked immunosorbent assay. d: HT29 cells were transfected with oligo RNA targeting RNF34 or control RNA. After 24 h, HT29 cells were transfected with siRNF34 or control siRNA again to increase the knockdown efficiency. After treatment with $\gamma$ TriDAP or vehicle, the lysates were blotting with NOD1 and GAPDH antibodies.

$\gamma$ TriDAP was used to activate endogenous NOD1, we found that the RNAi-specific knockdown of RNF34 expression increased the NF- $\kappa B$ luciferase activity and the production of IL-8 (Fig. 5B, 5C). Transfection of RNF34-specific siRNA, but not control siRNA, also enhanced the stability of NOD1 (Fig. 5D).

\section{Discussion}

NOD1 is expressed constitutively in intestinal epithelial cells where it helps to monitor the integrity of the cytosol and respond to the presence of enteric pathogens [19, 20]. Upon stimulation with peptidoglycan, NOD1 initiates its own oligomerization and exposes the CARD domain for recruitment and activation of receptor-interacting protein 2 (RIP2) via CARD-CARD interaction. Active RIP2 kinase can directly lead to ubiquitination of NEMO, which in turn activates the IKK complex and the subsequent phosphorylation of I $\kappa B$; NF- $\kappa B$ is then released and translocates to the nucleus. In the nucleus, NF- $\kappa B$ promotes the expression of various genes including those encoding pro-inflammatory cytokines, chemokines, growth factors, and others. Thus, NOD1 agonists are candidate adjuvants for the treatment of microbial infections that do not respond well to antibiotics, particularly for gastrointestinal and respiratory infections. However, increasing evidence has shown unexpected activation of NOD1 associated with tissue damage caused by both acute and chronic inflammation, such as septic shock and inflammatory bowel disease. Some NOD1 small-molecule antagonists, 
such as Noditinib-1 [21] and GSK'217 [22], have been developed to treat inflammatory diseases. Therefore, research on the regulation of this receptor will provide new insight into the molecular mechanism of inflammation, and may help in the search for new targets for treating inflammatory disease.

In the present study, we reported a novel NOD1 partner, RNF34, which was found by yeast two-hybrid screening. Interaction between RNF34 and NOD1 was further confirmed by GST pull-down and co-IP assays. Previously, RNF34 was identified as an E3 ligase that promotes the ubiquitin-mediated proteolysis of DED caspases [23]. Knockdown of RNF34 expression in tumor cell lines leads to apoptosis via increased activation of DED caspases. Wang et al. revealed that RNF34 is a cold-inducing E3 ubiquitin ligase for PGC-1 $\alpha$ and negatively regulates brown fat cell metabolism [24]. RNF34 was also reported to promote MDM2-independent p53 degradation [18].

Few negative regulators of NOD1 have been found to date. CENTB1 was the first molecule identified that directly and negatively regulates NOD1 activity [25]. In this report, the mechanism involved in the negative regulation and stability change of NOD1 by CENTB1 was not elucidated. Recently, PSMA7, another negative regulator, was reported to interact with NOD1 directly and promote the proteosome-dependent degradation and ubiqutination of NOD1. However, PSMA7 is not an E3 ligase, and therefore cannot ligate ubiquitin chains to NOD1 directly. Here, we report NOD1 as a novel substrate of RNF34. RNF34 is the first E3 ubiquitin ligase with demonstrated involvement in the ubiquitination of NOD1. NOD1 has been reported to be involved in activation of the NF- $\mathrm{KB}$ pathway through recognition of the intracellular bacterial component $\gamma$ TriDAP. To explore the functional role of these associations, NF- $\mathrm{\kappa B}$ activation was assessed in mammalian cells with overexpression or knocked down expression of RNF34. These experiments showed that RNF34 downregulates NF- $\kappa B$ activation via transfection of NOD1 plasmid or its ligands, whereas E3 ligase mutants lack this ability.

Thus, RNF34 might function to regulate host innate immune signaling by destabilizing NOD1. Therefore, approaches aimed at targeting RNF34, such as siRNA, could enhance these responses, which have broad implications for the treatment of inflammatory diseases.

\section{Acknowledgement}

This project is supported by Natural Science Foundation of Liaoning Province for Zhang R (Grant no. 2013020158).

\section{Reference}

1 Moreira LO, Zamboni DS: Nod1 and nod2 signaling in infection and inflammation. Front Immunol 2012;3:328.

2 Kumar H, Kawai T, Akira S: Pathogen recognition by the innate immune system. Int Rev Immunol 2011;30:16-34.

-3 Chamaillard M, Hashimoto M, Horie Y, Masumoto J, Qiu S, Saab L, Ogura Y, Kawasaki A, Fukase K, Kusumoto S, Valvano MA, Foster SJ, Mak TW, Nunez G, Inohara N: An essential role for nod1 in host recognition of bacterial peptidoglycan containing diaminopimelic acid. Nat Immunol 2003;4:702-707.

4 Hahn JS: Regulation of nod1 by hsp90 chaperone complex. FEBS Lett 2005;579:4513-4519.

5 Hasegawa M, Fujimoto Y, Lucas PC, Nakano H, Fukase K, Nunez G, Inohara N: A critical role of rick/rip2 polyubiquitination in nod-induced nf-kappab activation. EMBO J 2008;27:373-383.

6 Park JH, Kim YG, McDonald C, Kanneganti TD, Hasegawa M, Body-Malapel M, Inohara N, Nunez G: Rick/ rip2 mediates innate immune responses induced through nod1 and nod2 but not tlrs. J Immunol 2007;178:2380-2386.

7 da Silva Correia J, Miranda Y, Leonard N, Ulevitch RJ: The subunit csn6 of the cop9 signalosome is cleaved during apoptosis. J Biol Chem 2007;282:12557-12565. 


\section{Cellular Physiology $\quad$ Cell Physiol Biochem 2014;33:1954-1962 and Biochemistry

8 Girardin SE, Tournebize R, Mavris M, Page AL, Li X, Stark GR, Bertin J, DiStefano PS, Yaniv M, Sansonetti PJ, Philpott DJ: Card4/nod1 mediates nf-kappab and jnk activation by invasive shigella flexneri. EMBO Rep 2001;2:736-742.

9 Kim JG, Lee SJ, Kagnoff MF: Nod1 is an essential signal transducer in intestinal epithelial cells infected with bacteria that avoid recognition by toll-like receptors. Infect Immun 2004;72:1487-1495.

10 Zilbauer M, Dorrell N, Elmi A, Lindley KJ, Schuller S, Jones HE, Klein NJ, Nunez G, Wren BW, Bajaj-Elliott M: A major role for intestinal epithelial nucleotide oligomerization domain 1 (nod1) in eliciting host bactericidal immune responses to campylobacter jejuni. Cell Microbiol 2007;9:2404-2416.

11 Viala J, Chaput C, Boneca IG, Cardona A, Girardin SE, Moran AP, Athman R, Memet S, Huerre MR, Coyle AJ, DiStefano PS, Sansonetti PJ, Labigne A, Bertin J, Philpott DJ, Ferrero RL: Nod1 responds to peptidoglycan delivered by the helicobacter pylori cag pathogenicity island. Nat Immunol 2004;5:1166-1174.

12 Le Bourhis L, Magalhaes JG, Selvanantham T, Travassos LH, Geddes K, Fritz JH, Viala J, Tedin K, Girardin SE, Philpott DJ: Role of nod1 in mucosal dendritic cells during salmonella pathogenicity island 1-independent salmonella enterica serovar typhimurium infection. Infect Immun 2009;77:4480-4486.

13 Travassos LH, Carneiro LA, Ramjeet M, Hussey S, Kim YG, Magalhaes JG, Yuan L, Soares F, Chea E, Le Bourhis L, Boneca IG, Allaoui A, Jones NL, Nunez G, Girardin SE, Philpott DJ: Nod1 and nod2 direct autophagy by recruiting atg16l1 to the plasma membrane at the site of bacterial entry. Nat Immunol 2010;11:55-62.

14 da Silva Correia J, Miranda Y, Leonard N, Hsu J, Ulevitch RJ: Regulation of nod1-mediated signaling pathways. Cell Death Differ 2007;14:830-839.

15 da Silva Correia J, Miranda Y, Austin-Brown N, Hsu J, Mathison J, Xiang R, Zhou H, Li Q, Han J, Ulevitch RJ: Nod1-dependent control of tumor growth. Proc Natl Acad Sci U S A 2006;103:1840-1845.

16 Chen GY, Shaw MH, Redondo G, Nunez G: The innate immune receptor nod1 protects the intestine from inflammation-induced tumorigenesis. Cancer Res 2008;68:10060-10067.

17 Wang F, Jiang Z, Li Y, He X, Zhao J, Yang X, Zhu L, Yin Z, Li X, Wang X, Liu W, Shang W, Yang Z, Wang S, Zhen Q, Zhang Z, Yu Y, Zhong H, Ye Q, Huang L, Yuan J: Shigella flexneri t3ss effector ipah4.5 modulates the host inflammatory response via interaction with nf-kappab p65 protein. Cell Microbiol 2013;15:474-485.

18 Yang W, Rozan LM, McDonald ER, 3rd, Navaraj A, Liu JJ, Matthew EM, Wang W, Dicker DT, El-Deiry WS: Carps are ubiquitin ligases that promote mdm2-independent p53 and phospho-p53ser20 degradation. J Biol Chem 2007;282:3273-3281.

19 Keestra AM, Baumler AJ: Detection of enteric pathogens by the nodosome. Trends Immunol 2014;35:123130 .

20 Antosz H, Osiak M: Nod1 and nod2 receptors: Integral members of the innate and adaptive immunity system. Acta Biochim Pol 2013;60:351-360.

21 Correa RG, Milutinovic S, Reed JC: Roles of nod1 (nlrc1) and nod2 (nlrc2) in innate immunity and inflammatory diseases. Biosci Rep 2012;32:597-608.

22 Gatheral T, Reed DM, Moreno L, Gough PJ, Votta BJ, Sehon CA, Rickard DJ, Bertin J, Lim E, Nicholson AG, Mitchell JA: A key role for the endothelium in nod1 mediated vascular inflammation: Comparison to tlr4 responses. PLoS One 2012;7:e42386.

23 McDonald ER, 3rd, El-Deiry WS: Suppression of caspase-8- and -10-associated ring proteins results in sensitization to death ligands and inhibition of tumor cell growth. Proc Natl Acad Sci U S A 2004;101:61706175.

24 Wei P, Pan D, Mao C, Wang YX: Rnf34 is a cold-regulated e3 ubiquitin ligase for pgc-1alpha and modulates brown fat cell metabolism. Mol Cell Biol 2012;32:266-275.

25 Yamamoto-Furusho JK, Barnich N, Xavier R, Hisamatsu T, Podolsky DK: Centaurin beta1 down-regulates nucleotide-binding oligomerization domains 1- and 2-dependent nf-kappab activation. J Biol Chem 2006;281:36060-36070. 